\title{
How digital fabrication can enable new methods in the architectural practice?
}

\author{
Daniela Silva \\ ISCTE-IUL, Portugal \\ danielamoreirasilva@gmail.com \\ Alexandra Paio \\ ISCTE-Instituto Universitário de Lisboa, ISTAR-IUL, \\ Vitruvius FABLAB, Portugal \\ alexandra.paio@iscte.pt
}

\author{
José Pedro Sousa \\ Universidade do Porto, Faculdade de Arquitectura \\ + DFL/CEAU, Portugal \\ jsousa@arq.up.pt
}

\begin{abstract}
Design methods have always changed, considering that design thinking is bound to the representational medium. Thus, its scope can be expanded by the enlarged possibilities offered by the new media and methodologies. Today, the computational methods are a crucial medium for architectural design. Not only digital media is augmenting our design capacity in profound ways, but also digital fabrication is establishing itself as a core discipline for architecture. It has the power to link digital design information with physical production processes, thus opening the opportunity to materialize ideas, experiments and investigate physical or digital artifacts during the design process.
\end{abstract}

Keywords: Digital Fabrication; Processes; Methodologies; Design Studio.

\section{Introduction}

"(...) What can this medium do? (...) What do I wish to do with this medium? It matters that one works in a medium whose properties suit one's purposes: sometimes a more forgiving medium; sometimes a more rewarding medium; occasionally rigor for rigor's sake (...) Psychologists (and software experts) often employ the term "affordances" to describe the workable capacities of a medium. This reflects the truism that opportunities shape outlook: "how we see the world depends on what we can do whit it". (McCullough, 1998)

The rapid technological and social changes of the past two decades present complex challenges to architectural practice and is always of interest to seek an understanding of timespecific drivers and directions of change in practice (Kolarevic, 2003; Oxman, 2008; Corse, 2010). The evolution of the computational design methods has made the design studio a very different place than it was in the 90's (Hensel \& Nilsson, 2016). The speed of information exchange accelerated by digital technology has made architecture become fully global. Looking back, as Picon states $(2010,9)$ "the transformations that we are observing today are inseparable from conditions like globalization. They are also the result of a much longer and more complex historical process than the recent conversation of designers to digital tools". In this matter, the architecture field continues to adapt and change, faster than ever (Burry \& Burry, 2016). Collaborative production, where people have to coordinate with one another to get anything done, is considerably harder than simple sharing, but the results can be more profound. New tools allow large groups to collaborate, by taking advantage of non-financial motivations and by allowing for wildly differing levels of contribution.
Since then, a new generation of practitioners has emerged due to the creative use of advanced technologies. The computational methods are emphasizing on the process of design as a means through which projects are produced. The rigor of the thought process is as much as a part of design as making shapes, thus requiring the need to provide a detailed account of the nature of interaction with digital media and level of control the designer has over the process of design (Oxman, 2006; Rybczynski, 2011; Oxman, 2012). Much of the material world today, from the simplest consumer products to the most sophisticated airplanes, is created and produced using a process in which design, analysis, representation, fabrication and assembly are becoming a relatively seamless collaborative process that is solely dependent on digital technologies (Kolaveric, 2003).

The vast advancements brought by the information society at the end of the 20th century are unquestionably affecting all areas of knowledge (Gengnagel, 2015; Glynn \& Sheil, 2011). $A$ variety of digital tools are increasingly being used in the field of architecture during different stages of the project, accompanied by a rapid development rate of various software options (Steinberg, 2000). The application of robotics in construction for various production tasks is gaining an evergrowing application (Gramazio \& Kohler, 2014). The construction site of the future is going to look very different to the one we are used to today. Between drones, robotic bulldozers and 3D printers, architecture is learning how automation and robotics can be exploited on the site, making the scenarios starting to adapt to the technological solutions (Burry \& Burry, 2016; Menges, Sheil, Glynn \& Skavara, 2017). Consequently, the landscape of digital technologies in architectural practice since the beginning of the 21th century has deeply transformed protocols, design methodologies, and the conceptualization of the discipline. As Hansel \& Nilsson 
$(2016,1)$ states "the shape of practice is changing in interesting, pluralistic, productive and novel ways".

In this context, the digital fabrication can be defined as a new kind of industry that uses computer-controlled tools and processes to transform digital designs and materials directly into useful products. The use of digital fabrication as part of the design process may result in considerable productivity gains to the design process in the context of the design studio. The challenges are manifold, and these practices should be further explored because of their capacity to question the basis of education, practice, and the conceptualization of architecture.

The architectural practice has entered into a time in which digital design and fabrication technologies play an important role in the building work. In this age of digital fabrication, the idea of production can generate new architectural effects that are exposed through the intersection of form, material, and technique. The true potential of digital technologies is not to allow architects to make buildings more organic. Rather, these technologies bring the opportunity to allow architects to regain control over the construction process by presiding virtually over a series of assembly methods generally left to contracting counterparts in the field. Such control streamlines collaboration and encourages further iteration during the design phases of a project.

To clarify the meaning of this new challenge is necessary to understand the scope of these changes in the architecture practice. With this goal, this research examines the influence of the digital fabrication in the design methods of architectural practice, by analyzing three different international offices: (1) Sou Fujimoto that still resist to the use of digital tools; (2) Mark Burry, those prototypes as decision-making; and (3) and Snohetta, that simultaneously explores traditional handicraft and cutting edge digital technology.

To understand those transformations, this study is focused in understanding the implications of digital fabrication on architectural practice in the future, by positioning the use of digital fabrication not as a tool, but as design thinking.

\section{Case Studies}

To answer the paper question, three offices practices were selected as case studies. They were selected for their specific relations with the use of digital technologies in their creative process and their unique perspective of the use of the digital fabrication in their methodologies. The offices were studied and analyzed following criteria such as project scale, representation, generation, design processes, techniques employed. The objective is to compare and contrast findings in order to identify similarities and/or differences.

The methodology used consists in interviews with the architects, observation of the work development and analysis of the office's projects, in order to understand the relationship between tools, office methodology, final result (project) and the extent of the use made by digital fabrication. The main purpose is to question each office if the digital fabrication has come as a tool to support the existing process of thinking, or as a new methodology, that is changing their way of approaching a project.

To understanding different design methods resulting from specific relations with digital technologies in our contemporaneity, the following design studios are selected to support a critical analysis and comparison.

Figure 1: Early sketch for the project Serpentine Gallery Pavilion 2013, by Sou Fujimoto Architects.

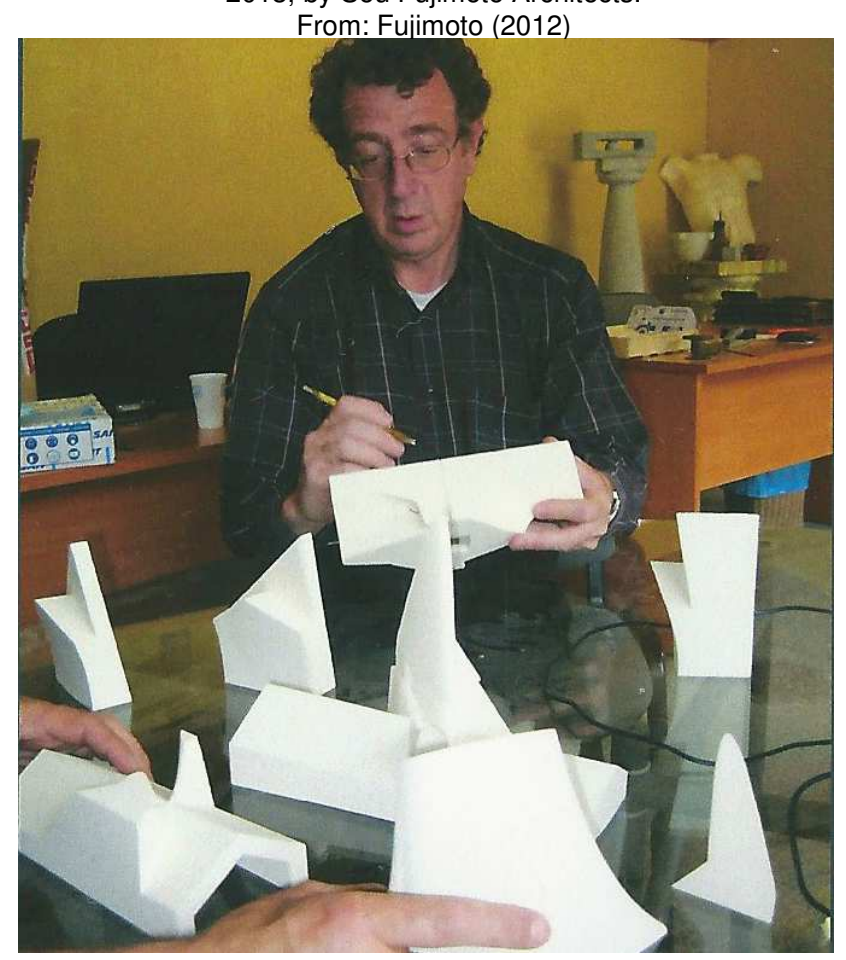

Figure 2: The large scale model (in this case 1:10 3D printed gypsum plaster rapid prototype) is the most effective communication device between designer and stonemason. From: Burry (2008) 


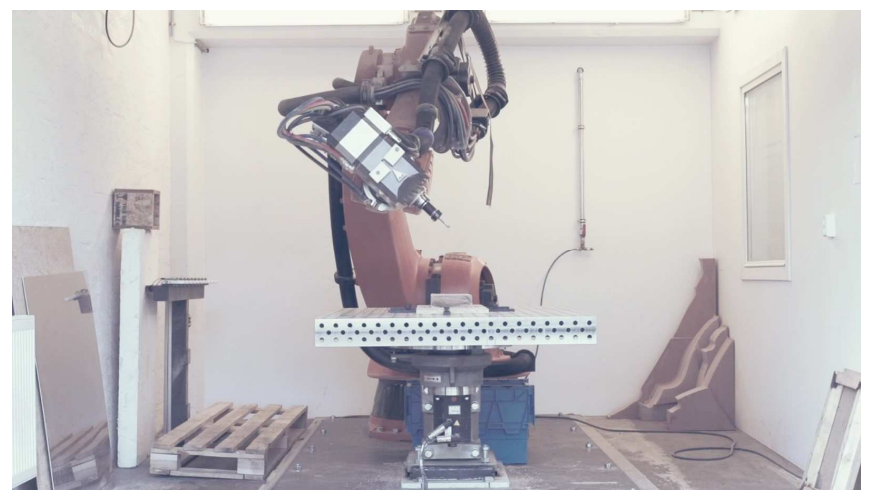

Figure 3: At the core of the design studio is a state-of-the art modeling workshop equipped with 3D rapid prototyping capabilities and a large, programmable manufacturing robot.

From: http://snohetta.com/process/design-methodology

\section{Sou Fujimoto: Resisting to the use of digital tools}

Sou Fujimoto is a young Japanese architect that stands for unconventional buildings that cannot be described by standard criteria. He's part of a new generation of architects that is forcing their way onto the stage in Japan. Whereas digital, parametric architecture has become the standard everywhere else in the world, in Japan there has emerged, almost imperceptibly, a generation of young architects who are setting off on their own paths, unaffected by it. The sketch is a way to reflect dialogue and discover. He sees the hand as the link between idea and result, and when you adopt a machine as a design process, you break this direct link (Figure 1).

The main question here, is why a young architect as Sou Fujimoto resists using digital tools as a design process? In an era where people with an architecture education are focusing on areas outside the field of architecture, he has defiantly clung to architecture. Fujimoto has turned to the roots of architecture as one of the oldest technologies from the far distant past. But he does it not as a simple revival of the past. But because his designs do not rely on cutting-edge technology, Fujimoto's architecture could have also existed in the past.

The quickest way to envisage an imagined idea is by hand. For Sou Fujimoto the act of sketching is like a dialogue with himself. His design process is based on sketch and physical modeling, as a way to reflect, dialogue and discover. For him, a drawing translates and visualizes thoughts with unique, inimitable immediacy and a model represents them. In both of them - this is my interpretation after my experience working at his office - the hand is the link between the idea and the result. The moment that you adopt a machine as a design process, you break this direct link and become something else. When he puts pen to paper, there a lack of conscience. He does not yet know what it is, nor what will be drawn. Within the transition of some unknown lines into a shape, a sketch is born. The same with the physical model.

\section{Mark Burry: Prototypes to decision-making}

Mark Burry work in-practice is focused on Gaudi's legacy at the Sagrada Família, by following his definitive plaster models for the project, his use of geometry and his architecture. The interest on Burry's work is focused on Gaudi's approach to design, his subsequent path to construction, and how relevant is it now when we look at how the Sagrada Família Church continues today using digital aids. His task in the Sagrada Familia Church was possible by using the most advanced technology, as much for the production of drawings and plaster models as for advancing the construction.

Mark Burry's work was made possible thanks to the profound research into Gaudi's original project and the use being made of the most advanced technology, as much for the production of drawings and plaster models as for advancing the construction, is an interesting contribution to architectonic culture today. Gaudi was in a constant quest to stretch the limits of architecture, but almost nothing is revealed about his design process. His techniques and construction process are admired for the innovation that they reveal, especially in our post-digital era. It has only been in recent years that the Sagrada Família Church project has emerged from its position as an assumed chronological error to being recognized as one of the world's most coherent architectural projects in a postdigital era.

When Burry picked up Gaudi's work, it soon became clear that conventional architectural software was no use at all in dealing with such complex geometries, and from that point on, there was a need to adopt more advanced technologies. Aeronautical design software and cutting-edge software and hardware came as the perfect answer to the challenges ahead.

With the digital era and all the possibilities that came with it, the Sagrada Família Church technical office and its collaborating universities were quick to understand the opportunities it created. The early recognition of the potential of digital models has naturally meant that the Sagrada Família Church was one of the first projects anywhere to experiment with rapid prototyping. Today the design process hinges on both the gypsum plaster model and the drawing, as well as on the virtual model and the 3D printed rapid prototype (Figure 2). They are taking advantage of the speed and accuracy of digital techniques, but the sketch usually prevails as the most effective interface between the various protagonists to understand and communicate the project solutions.

A parametrical model, is nothing more than a digital model that allows us to interact and transform freely. Gaudi was already working on these flexible models, but not in a digital way. The famous hanging model made of little bags of birdshot linked between them with fine cord was just that: a flexible model to be played with. He used and manipulated this model to discover the final design of the church's structure. With the arrival of digital modeling and the geometrical complexity of the project, the Sagrada Família Church took advantage of several software manufacturers who sponsored the evaluation of this software in an architectural project, making it the first architectural projects to make use of the parametric design. At 
the beginning the software was helping with the understanding of Gaudi's engineering, but later it was a powerful tool on the design process. Thanks to this process, they were reaching the desired outcome more quickly and more deeply than could otherwise be possible.

The commitment of Mark Burry with the project and the need to answer the project complexity, made him move the office to the construction site. After all, in this case there is no separation between what is the architect role and the construction site. The boundary between the two is blurred and everything happens on the site, drawings, study models, construction, etc. Proving that the paradigm of the processes in architecture is changing. No longer can the architect hide his responsibility in the drawings that he produces.

\section{Snohetta: Prototyping processes}

Their working method simultaneously explores traditional handicraft and cutting edge digital technology, becoming a complementary relationship in the creative process (Figure 3).

At the core of the design studio is a state-of-the art modeling workshop equipped with 3D rapid prototyping capabilities and a large, programmable manufacturing robot. Alongside traditional woodworking machines, these tools enable rapid prototyping to become an integral part of the design process. This way, ideas can move seamlessly between analogue and digital worlds. Workshops and tools can only go so far, since people drive the creative process.

Snøhetta practices a self-defined trans-disciplinary process in which different professionals - from architects to visual artists, philosophers to sociologists - exchange roles in order to explore differing perspectives without the prejudice of convention. They believe that the step from advanced geometry to fabrication is still one of the largest challenges we face in design today. They explore advanced technologies both in the design and the fabrication process by combining a wide spectrum of tools and theories that leads to a continuous state of reinvention.

\section{Preliminary results}

The overall objective of this study, as a part of major ongoing research, is to assess and promote the potential of digital fabrication as a methodology in the architectural practice context and to clarify how digital fabrication is envisioned to lead to a radical paradigm shift in the design process. The hypothesis states that the use of digital fabrication in the development of a project is problem solving and have profoundly enhanced creativity in traditional architectural methodologies. Generally speaking, architectural practices are actively embracing digital tools and methodologies as a strategy in the project development. Design studios lead this transformation by pushing design innovation as a core objective in their proposals. Many architectural offices have established internal research units to further investigate their insertion into practice.
The role of Sou Fujimoto in the architecture panorama is very much linked to the idea of the architect as the artist, by having a dialog between his hand drawings and the constructed building. By doing so, he denies any sort of digital mythology as design process. Mark Burry's work presents and explores new opportunities for the current evolution of architecture by means of digital technologies. On the other hand, Snohetta combine technology with traditional. 3D rapid prototyping and manufacturing robot are at the center of their workshop, but they defend that workshops and tools can only go so far, since people drive the creative process, outlining people from the machine in the design process.

However, none of them are using the tool as new design thinking practice. The use of digital fabrication as part of the design process early stages, if pursued with a rational and selective approach, may result in considerable productivity gains to the design process in the context of the design studio. But is this all? A tool depends on us to control the scope, the pace, and the focus of its work; merely attended machinery does not (McCullough, 1998).

\section{Towards an answer}

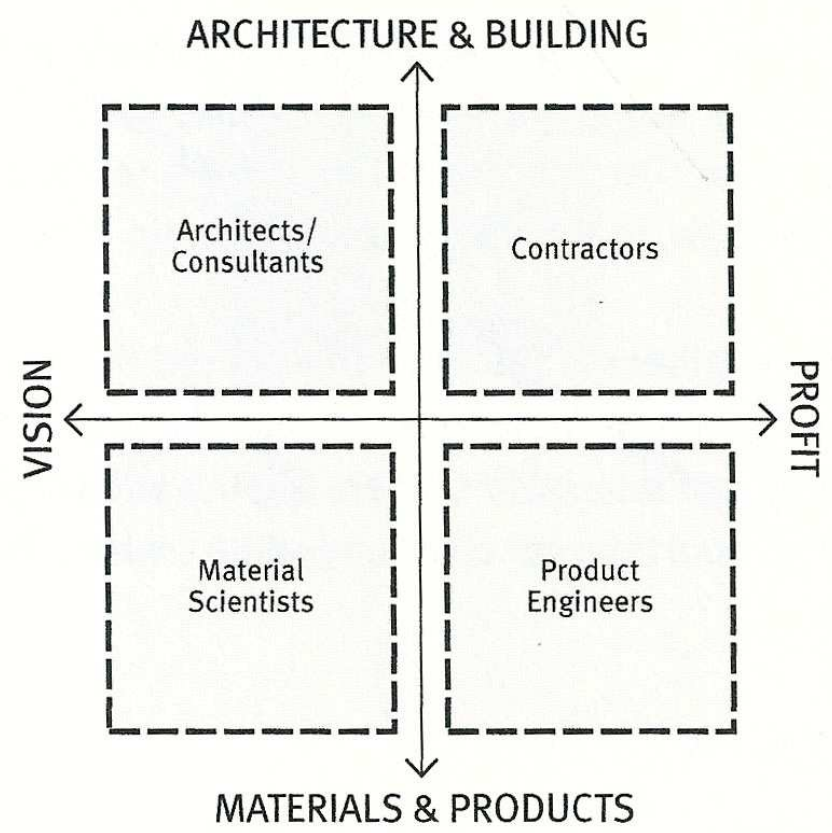

Figure 4: The stratification and segregation of architecture. From: Kieren and Timberlake, 2004 


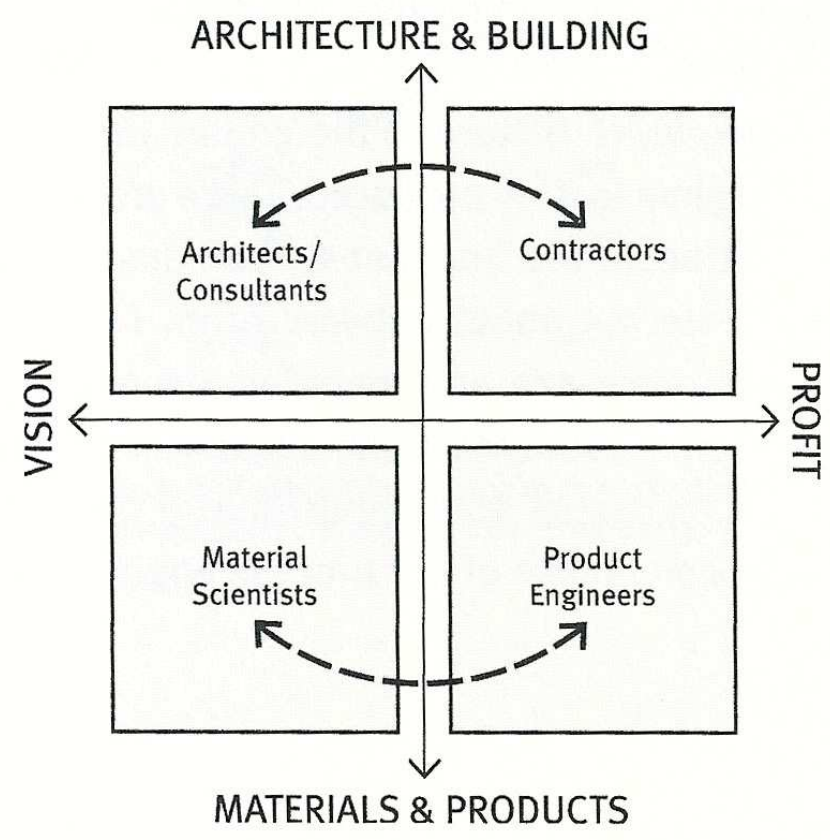

Figure 5: Limited communication. From: Kieren and Timberlake, 2004

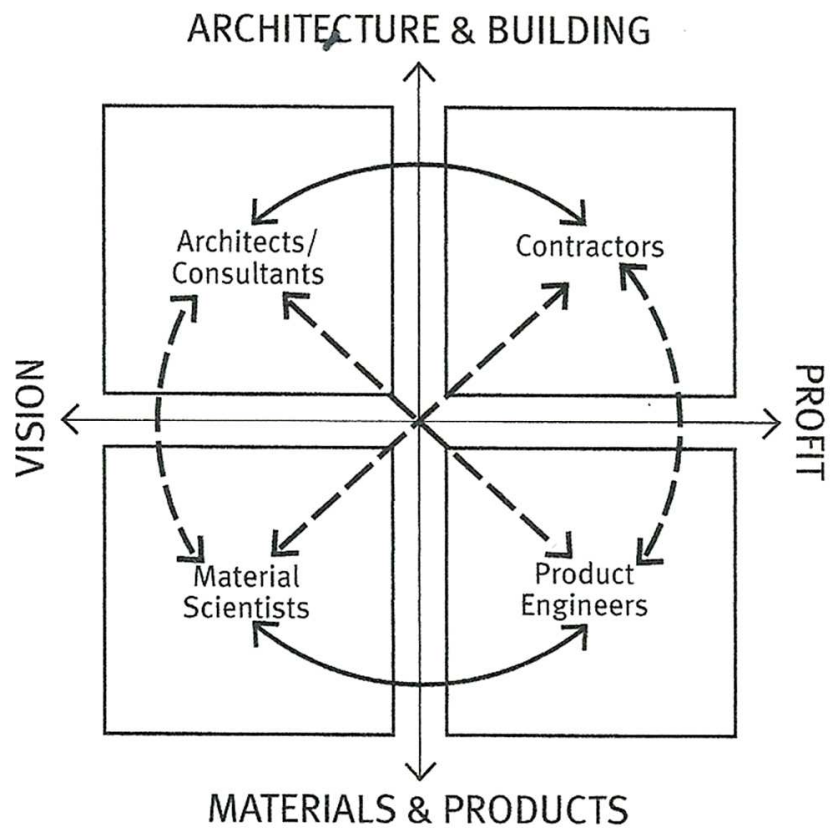

Figure 6: Breaking out of the box. From: Kieren and Timberlake, 2004

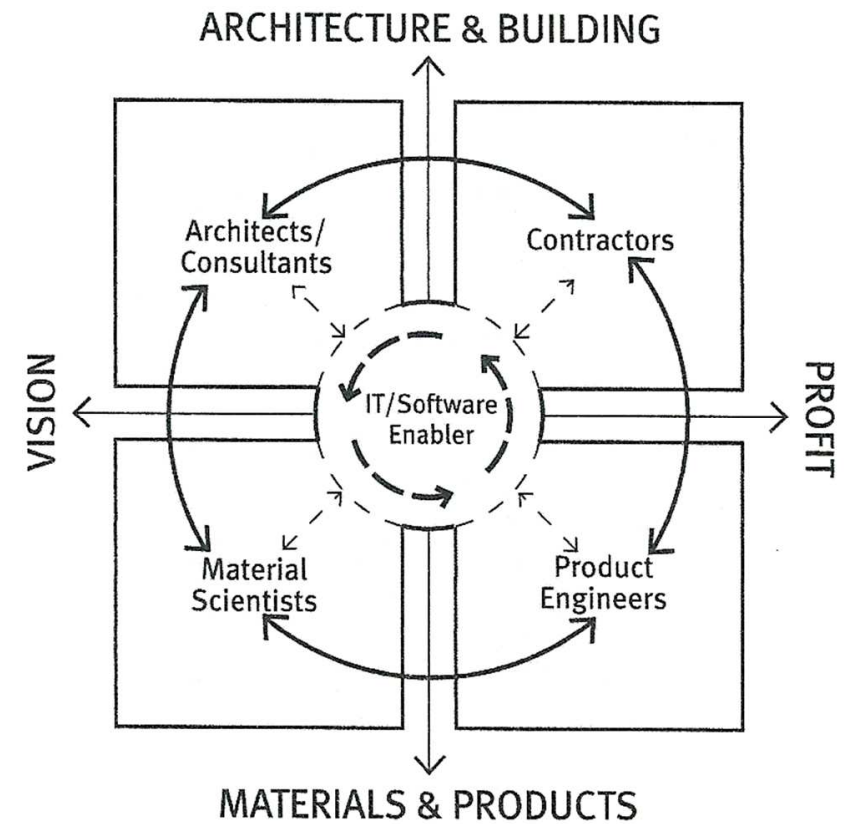

Figure 7: Enabling collective intelligence.

From: Kieren and Timberlake, 2004

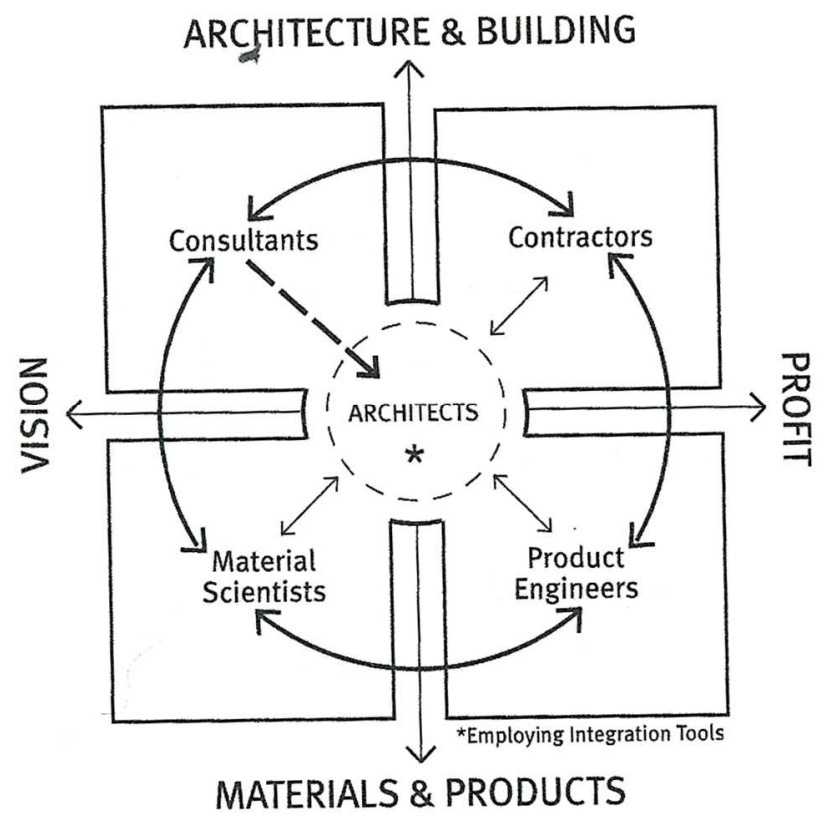

Figure 8: Managing intelligence. From: Kieren and Timberlake, 2004 
Design is a creative and hybrid activity, which depends on a proper blend of arts, science and mathematics for its successful execution. Although there are various definitions of the term creativity, most definitions imply the development of something new. "Creativity is often thought of as the ability to generate ideas that are both innovative and functional" (Alomar, 2000). As a consequence of the modernism, there has been segregation between the designers and makers. The architect has been separated from the contractor. Industries like automotive, aircraft and shipbuilding have developed models of engagement that integrate all acts of design and production. They have already implemented the tools of the new collective intelligence paradigm. Designers and producers are members of a team that comes together to solve specific problems.

As Kieran and Timberlake stated, the relations among those responsible for the making of things, has been redesigned. In their statement, the authors identify the four major actors in the architectural production (architects/consultants, contractors, material scientists and product engineers) and they explain different types of collaboration and consequently, of relationship, between them. They describe the first type of collaboration as "the stratification and segregation of architecture" (Figure 4). Current architectural production is typified by stratification of the various components used in designing and implementing a building. As a result, the corresponding disciplines that are responsible for each segregate themselves within the stratified field. There is total self-segregation and no collective intelligence. The second type, they call it "limited communication" (Figure 5). At this point, there is very little communication in the architectural model between disciplines. What communication does exist is not a true communicative relationship, but rather a hierarchical one in which one party is hired by the other to fulfill a particular role, such as a lighting consultant providing lighting scheme for an architect.

The "breaking out of the box" (Figure 6) is the need that the four major disciplines have to cross the boundaries established by their traditional roles. All parties must seek a balance between vision and profit. There need to be reciprocal relationships between the developers of materials and products with the implementers and appliers.

Thanks to an entire new industry that produces communication/collaboration software, it was possible for the various parties involved in a project to have real-time sharing of information, in this sense "enabling collective intelligence" (Figure 7). This instantaneous communication allows each party to be aware and involved with the other various disciplines throughout the entire process of a project. And the last one they called it "managing intelligence" (Figure 8). Architects will serve as the overseers of the exchange of information. They will orchestrate the interactions and prompt the disciplines to work together. This role is not advocacy of the architect as a master builder, but rather as a twenty-first century maestro.

The processes transformation that we do not see represents a profound change in the way we make things. By positioning the use of digital fabrication in the different phases of the design process as a support to the architect work, it is presented a new methodology. Generally speaking, architects keep using digital fabrication as a tool to produce a final object, a model. However, there are some architectural practices that are starting to explore the possibilities enabled by digital fabrication with a design process that incorporates the full range of digital technology. Digital tools in architectural practice replace the art of drafting with software that simulates the representational orthographic standards of production. The majority (if not all professional software) are used to represent the building design. It is this production standard in practice that is beginning to be challenged by new digital tools. Designers use media, design environments, not as a passive mechanism, but as a way to inform their design.

The design process involves a variety of different types of thinking. There are few revolutions in the making of things. Change is almost always a matter of gradual movement from one manner of making and appearance to another. Change in the making of architecture, in its unseen processes, is already here (Kieran and Timberlake 2004). The study outcomes reveal that design studio methodologies are becoming highly interactive and fast-paced and digital crafting is challenging the practices of design in architecture in many ways. Digital practices are actively providing new methodological strategies that are deeply affecting the depth, the relevance, and the emphasis of each stage during the design process. Furthermore, they may be profoundly transforming the conceptualization of the architectural practices for this new century. The use of digital tools has proved to be highly significant during all project stages: analysis, design and representation. To identify and propose suitable digital design tools for implementation in the context of current or future architectural design studios seems to be critical, as it establishes new workflows. The maturing of interfaces between the design space of the architect and the production space of the manufacturer is leading to the shaping of new methodologies.

In opposition to these statements, Sou Fujimoto example comes to the equation to counterbalance with his approach on the design process. In the sense that his role in the architecture panorama is very much linked to the idea of the architect as the artist, by having a dialog between his hand drawings and the constructed building. By doing so, he denies any sort of digital mythology as design process. Several digital tools currently available for architectural purposes are explored with the objective to identify and propose their suitable implementation in current and future architectural design studios. But it's time to understand how they might affect design methodologies, as well as identifying problems, challenges, and potentials.

Architects should go beyond thinking about the formal output of their designs using new digital tools and actually seek to uncover the main purpose of design and digital fabrication. The process of making is no longer entirely linear. Producers engage in design, and designers engage in production. Digital fabrication helps the process since the production has become part of the design process by working with assemblers from 
the outset. Designers picture how things are made, their sequence of assembly and their joining systems and digital fabrication combines all the actors in the process and helps them to test and communicate the designs, making the process totally collaborative.

Processes, materials and information tools are present today in ways that have not been integrated in high architecture since the Renaissance. Architects can no longer claim exclusion on the basis of attachment to the ground and customized design and construction. Digital fabrication as a methodology in the design studio it is exciting, as it is the wave of the future. New processes offer new opportunities to architecture: more control, higher quality and improved features. To do so, we must look deeper into what lies beyond how things look like, and to see how we do things.

\section{References}

Burry, M. and Bonet, J. (2008) - Gaudi Unseen, Jovis;

Burry, M. and Burry, J. (2016) - Prototyping for Architects, Thames \& Hudson, London;

Corse, R. (2010) - Fabricating Architecture: Selected Readings in Digital Design and Manufacturing, Princeton Architectural Press, New York;

Fujimoto, S. (2012) - Sou Fujimoto: Sketchbook, Lars Müller;

Gengnagel, C. (2015) - Rethinking Prototyping- Transdisciplinary Concepts of Prototyping, Springer International Publishing, Berlin;
Glynn, R. and Sheil, B. (2011) - Fabricate: Making Digital Architecture, Riverside Architectural Press;

Gramazio, F. and Kohler, M. (2014) - The Robotic Touch. How Robots Change Architecture, Park Books, Zurich;

Hansel and Nilsson, (2016) - The Changing Shape of Practice: Integrating Research and Design in Architecture, Routledge;

Kieren, S. and Timberlake, J. (2004) - Refabricating Architecture: How Manufacturing Methodologies are poised to Transform Building Construction, McGraw-Hill Education;

Kolarevic, B. (2003) - Architecture in the Digital Age: Design and Manufacturing, Spon, New York;

McCullough, M. (1998) - Abstracting Craft: The Practiced Digital Hand, The MIT Press;

Menges, Sheil, Glynn and Skavara, (2017) - Fabricate 2017: Rethinking Design and Construction, UCL Press;

Oxman, R. (2006) - Theory and design in the first digital age, Design studies 27;

Oxman, R. (2008) - Digital Architecture as a challenge for design pedagogy: theory, knowledge, models and medium, in Design Studies, pp. 99-120;

Picon, A. (2010) - Digital Culture in Architecture. An Introduction for the design professions, Birkhäuser, Basel;

Steinberg, M. (2000) - New Technologies in Architecture - Digital Design and Manufacturing Techniques, Harvard University Press, Cambridge, pp. 74-75. 